EPJ Web of Conferences 73, 06007 (2014)

DOI: $10.1051 /$ epjconf/20147306007

(C) Owned by the authors, published by EDP Sciences, 2014

\title{
Dispersion relations in application to chiral two-nucleon dynamics
}

\author{
A.M. Gasparyan ${ }^{1,2, a}$, M.F.M. Lutz ${ }^{3}$ and E. Epelbaum ${ }^{1}$ \\ ${ }^{1}$ Institut für Theoretische Physik II, Fakultät für Physik und Astronomie, Ruhr-Universität Bochum \\ 44780 Bochum, Germany \\ ${ }^{2}$ SSC RF ITEP, Bolshaya Cheremushkinskaya 25, 117218 Moscow, Russia \\ ${ }^{3}$ GSI Helmholtzzentrum für Schwerionenforschung GmbH, Planckstraße 1, \\ 64291 Darmstadt, Germany
}

\begin{abstract}
A novel scheme based on the chiral Lagrangian is applied to the nucleon-nucleon interaction close to threshold. Subthreshold partial-wave amplitudes are calculated in chiral perturbation theory and analytically extrapolated above threshold. The constraints imposed by analyticity and unitarity are used to stabilize the extrapolation. A reasonable description of the empirical phase shifts up to laboratory energies $T_{\text {lab }} \simeq 300 \mathrm{MeV}$ is obtained in terms of the parameters relevant at next-to-next-to-next-to-leading order. The convergence properties of the method and the comparison with the conventional potential approach are discussed.
\end{abstract}

\section{Introduction}

Chiral perturbation theory provides a systematic tool for studying low-energy hadron dynamics. The perturbative expansion works well in particular in describing the $\pi \pi$ and $\pi N$ scattering processes in the threshold region. On the other hand in the nucleon-nucleon sector a strict perturbative approach is unable to describe the existence of the bound and quasi-bound state close to threshold. A solution of this problem was suggested by Weinberg [1]. The scattering amplitude is generated by solving a dynamical equation in a non-perturbative manner with a potential calculated from the chiral Lagrangian. Within this scheme significant progress has been achieved in the recent years $[2,3]$.

There exists an alternative powerful approach based on analytic properties of the scattering amplitude [4]. It relies on the assumption that the interaction becomes perturbative in some energy region below the two-nucleon threshold. Such a behavior is known from quantum-mechanical two-body potential scattering even for rather strong interaction between two particles. It was demonstrated e.g. in Ref. [5] by the toy model example of the Yukawa potential. The method was worked out in detail for the case of $\pi N$ [4] and $\pi \pi$ [6] scattering and processes involving photons $[4,7,8]$ and turned out to be quite successful in the sense that one is able to describe experimental data rather well with a small

\footnotetext{
ae-mail: ashot.gasparyan@rub.de
}

This is an Open Access article distributed under the terms of the Creative Commons Attribution License 4.0, which permits unrestricted use, distribution, and reproduction in any medium, provided the original work is properly cited. 


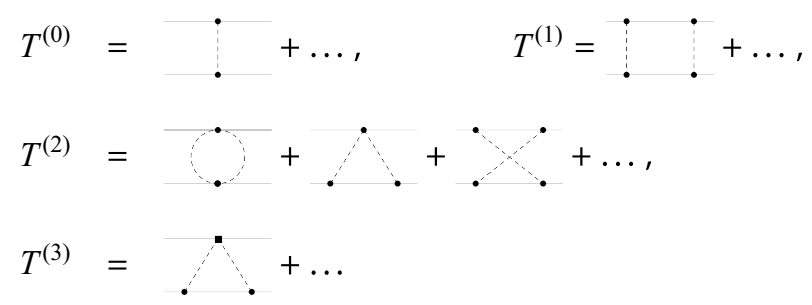

Figure 1. Feynman diagrams contributing to the NN scattering amplitude $T^{(i)}$ at order $Q^{i}$ in the chiral expansion. Solid dots (filled squares) denote the lowest-order vertices in the chiral Lagrangian (subleading vertices $\propto c_{i}$ ). The ellipses denote diagrams leading to shorter-range contributions.

number of parameters and to achieve a reasonable convergence of the theory. Recently, it was applied to the $N N$-scattering problem [9], see also Ref. [10-12] for related works.

\section{Partial wave dispersion relation}

The analytic structure of the nucleon-nucleon partial-wave amplitude $T(s)$ as a function of Mandelstam variable $s$ can be described in terms of the dispersion relation

$$
T(s)=U(s)+\int_{4 m_{N}^{2}}^{\infty} \frac{d s^{\prime}}{\pi} \frac{s-\mu_{M}^{2}}{s^{\prime}-\mu_{M}^{2}} \frac{T(s) \rho\left(s^{\prime}\right) T^{*}\left(s^{\prime}\right)}{s^{\prime}-s-i \epsilon},
$$

where the discontinuity along the right-hand cut (coming from the integral on the r.h.s. of Eq. (1)) is given by the $s$-channel unitarity. On the other hand, the generalized potential $U(s)$ possesses only the left hand cuts (we neglect the contributions from the inelastic channels) and can be represented as [4]

$$
U(s)=U_{\text {inside }}(s)+U_{\text {outside }}(s),
$$

where $U_{\text {inside }}(s)$ is calculated in chiral perturbation theory through the discontinuity $\Delta T(s)$

$$
U_{\text {inside }}(s)=\int_{\Lambda^{2}}^{4 m_{N}^{2}-M_{\pi}^{2}} \frac{d s^{\prime}}{\pi} \frac{\Delta T\left(s^{\prime}\right)}{s^{\prime}-s},
$$

whereas $U_{\text {outside }}(s)$ is determined in the low-energy domain $\Omega$ using conformal mapping techniques [4]. This domain includes the energy region we are interested in and defines the separation of the 'inside' and 'outside' contributions to the generalized potential. Outside of the $\Omega$ region the generalized potential $U(s)$ cannot be calculated reliably. This is in particular for either $s>\Lambda_{s}^{2}$ or $s<\Lambda^{2}$. We choose $\Lambda^{2}=4 m_{N}^{2}-9 M_{\pi}^{2}$ at the beginning of the $3 \pi$-cut separating the nonperturbative short range dynamics and $\Lambda_{s}^{2}=\left(2 m_{N}+2 M_{\pi}\right)^{2}$ corresponding to energies where inelastic channels become important.

Finally, one restores the amplitude above the threshold by solving Eq. (1) with $U(s)$ as an input. By construction, the amplitude $T(s)$ coincides with its perturbative expansion at the matching point $\mu_{M}$ where ChPT is assumed to converge most rapidly. Its exact position is not important but it must be sufficiently far away from $s$ - and $t$-channel unitarity cuts (excluding the one-pion exchange cut). We choose it, therefore, to be $\mu_{M}^{2}=4 m_{N}^{2}-2 M_{\pi}^{2}$. The diagrams that contribute to the amplitude at different orders in ChPT are shown in Fig. 1 excluding short-range terms containing contact interactions. Note that the amplitudes at the matching point and the discontinuity of the generalized potential are calculated in a manifestly covariant framework. The renormalization of the loop diagrams is performed using the strategy similar to the ones proposed in [13-15]. 

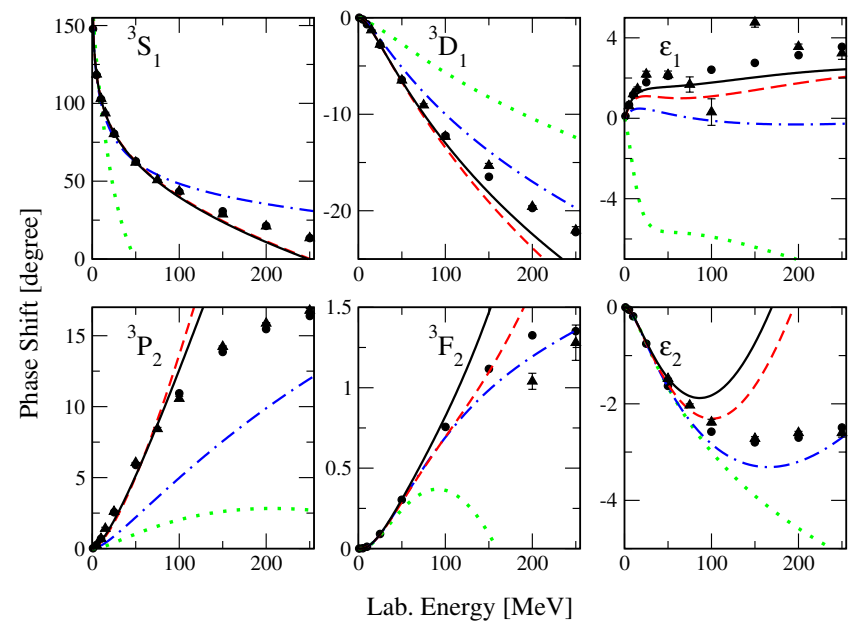

Figure 2. Phase shifts and mixing angles in the coupled ${ }^{3} S_{1}-{ }^{3} D_{1}$ and ${ }^{3} P_{2}-{ }^{3} F_{2}$ channels calculated at orders $Q^{0}$ (dotted lines), $Q^{1}$ (dash-dotted lines), $Q^{2}$ (dashed lines) and $Q^{3}$ (solid lines) in comparison with the Nijmegen [16] (filled circles) and SAID [17] (filled triangles) PWAs.

Table 1. Generalized potential at the matching point $U\left(\mu_{M}^{2}\right)$ at different chiral orders in the uncoupled channels (after subtracting the dominant one-pion exchange contribution).

\begin{tabular}{|c|c|c|c|c|}
\hline & ${ }^{1} S_{0}$ & ${ }^{1} P_{1}$ & ${ }^{3} P_{1}$ & ${ }^{3} P_{0}$ \\
\hline$Q^{0}$ & $5.79 \times 10^{2}$ & 0 & 0 & 0 \\
\hline$Q^{1}$ & $5.82 \times 10^{2}$ & $4.91 \times 10^{3}$ & $4.33 \times 10^{3}$ & $12.41 \times 10^{3}$ \\
\hline$Q^{2}$ & $8.53 \times 10^{2}$ & $1.42 \times 10^{3}$ & $3.46 \times 10^{3}$ & $-6.43 \times 10^{3}$ \\
\hline$Q^{3}$ & $8.63 \times 10^{2}$ & $2.19 \times 10^{3}$ & $4.81 \times 10^{3}$ & $-5.70 \times 10^{3}$ \\
\hline
\end{tabular}

Table 2. Matrix elements of the generalized potential at the matching point $U_{i j}\left(\mu_{M}^{2}\right)$ at different chiral orders in the coupled channels (after subtracting the dominant one-pion exchange contribution).

\begin{tabular}{|c|c|c|c|}
\hline & ${ }^{3} S_{1}-{ }^{3} D_{1}, U_{11}$ & ${ }^{3} S_{1}-{ }^{3} D_{1}, U_{12}$ & ${ }^{3} P_{2}-{ }^{3} D_{2}, U_{11}$ \\
\hline$Q^{0}$ & $-2.95 \times 10^{2}$ & 0 & 0 \\
\hline$Q^{1}$ & $6.14 \times 10^{2}$ & $6.70 \times 10^{3}$ & $2.44 \times 10^{2}$ \\
\hline$Q^{2}$ & $2.08 \times 10^{2}$ & $-5.92 \times 10^{3}$ & $5.59 \times 10^{2}$ \\
\hline$Q^{3}$ & $2.07 \times 10^{2}$ & $-6.94 \times 10^{3}$ & $7.50 \times 10^{2}$ \\
\hline
\end{tabular}

\section{Results}

The free parameters of the effective theory determine the coefficients of the conformal expansion. Such low-energy constants are adjusted to the empirical $N N$ phase shifts. We consider only the lowest ( $S$ and $P$ ) partial waves where the non-perturbative dynamics is most prominent. We fit to the energy points below $T_{\mathrm{lab}}=100 \mathrm{MeV}$ in order to correctly reproduce the threshold physics. The results for the coupled partial waves are shown in Fig. 2. The quality of the obtained fit is comparable with the one in the potential approach (at next-to-next-to-leading order). The convergence pattern from the order $Q^{0}$ to $Q^{3}$ appears very reasonable. This supports our assumption of the perturbative nature of the nucleon-nucleon interaction in the subthreshold region.

A more direct indication of convergence of the perturbation theory below threshold are the values of the partial wave amplitudes at the matching point $s=\mu_{M}^{2}$ (see Tables 1,2). Note, however, that in order to draw an ultimate conclusion about convergence one should specify the renormalization scheme. The details of the renormalization for the $N N$ amplitude will be given in a forthcoming publication. 


\section{Summary}

The nucleon-nucleon interaction was studied from threshold up to $T_{\text {lab }}=250$. A novel approach that constructs an analytic extrapolation of subthreshold amplitudes, that are calculated in ChPT, was applied. It relies on using partial-wave dispersion relations properly combined with conformal mapping techniques. The free parameters of the chiral Lagrangian were adjusted to the nucleon-nucleon phase shifts using existing partial-wave analyses. The obtained fits are in a reasonable agreement with the empirical PWAs. The quality of the fit at order $Q^{3}$ is comparable with the one obtained in conventional approaches based on the chiral expansion of the NN potential. The expansion for the phase shifts converges when going from the order $Q^{0}$ to $Q^{3}$ which supports our assumption of the validity of a perturbative expansion of the $\mathrm{NN}$ amplitude in the subthreshold region. Another indication of convergence of the chiral expansion below threshold is given by the calculation of the amplitude at the matching point at different orders.

This work is supported by the EU HadronPhysics3 project "Study of strongly interacting matter", by the European Research Council (ERC-2010-StG 259218 NuclearEFT).

\section{References}

[1] S. Weinberg, Phys. Lett. B 251, 288 (1990)

[2] D. Entem, R. Machleidt, Phys. Rev. C 68, 041001 (2003), nucl-th/0304018

[3] E. Epelbaum, W. Glöckle, U.-G. Meißner, Nucl.Phys. A 747, 362 (2005), nucl-th/0405048

[4] A. Gasparyan, M.F.M. Lutz, Nucl. Phys. A 848, 126 (2010), 1003. 3426

[5] I. Danilkin, A. Gasparyan, M. Lutz, Phys. Lett. B 697, 147 (2011), 1009.5928

[6] I. Danilkin, M. Lutz, EPJ Web Conf. 37, 08007 (2012), 1208. 2568

[7] A. Gasparyan, M. Lutz, B. Pasquini, Nucl. Phys. A 866, 79 (2011), 1102. 3375

[8] I. Danilkin, M. Lutz, S. Leupold, C. Terschlusen, Eur. Phys. J. C 73, 2358 (2013), 1211.1503

[9] A. Gasparyan, M. Lutz, E. Epelbaum, Eur. Phys. J. A 49, 115 (2013), 1212 . 3057

[10] M. Albaladejo, J. Oller, Phys. Rev. C 84, 054009 (2011), 1107. 3035

[11] M. Albaladejo, J. Oller, Phys. Rev. C 86, 034005 (2012), 1201.0443

[12] Z.H. Guo, J. Oller, G. Rios (2013), 1305.5790

[13] M. Lutz, Nucl. Phys. A 677, 241 (2000), nucl-th/9906028

[14] A. Semke, M. Lutz, Nucl. Phys. A 778, 153 (2006), nucl-th/0511061

[15] T. Fuchs, J. Gegelia, G. Japaridze, S. Scherer, Phys. Rev. D 68, 056005 (2003), hep-ph/0302117

[16] V. Stoks, R. Kompl, M. Rentmeester, J. de Swart, Phys. Rev. C 48, 792 (1993)

[17] R. Arndt, W. Briscoe, I. Strakovsky, R. Workman, Phys. Rev. C 76, 025209 (2007), 0706.2195 